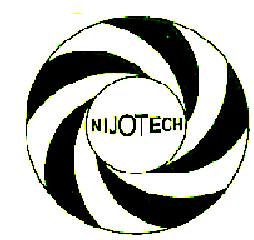

Nigerian Journal of Technology (NIJOTECH)

Vol. 33. No. 1. January 2014, pp. 72 - 77

Copyright@ Faculty of Engineering,

University of Nigeria, Nsukka, ISSN: $1115-8443$ www.nijotech.com

http://dx.doi.org/10.4314/njt.v33i1.10

\title{
APPLICATION OF INTEGRATED MODELING TECHNIQUE FOR DATA SERVICES RESOURCE ALLOCATION IN ATM BASED PRIVATE WAN
}

\author{
F. I. Onah ${ }^{1}$, C. I Ani'2,* \\ 1National Communications Commission, Abuja, NIGERIA \\ 2 ETF CENTRE of EXCELLENCE IN NETWORKING, DEPT. of ELECTRONIC ENGR'G, UNiv. OF NigERIA, NSUKKA, NIGERIA. \\ E-mail addresses:1 fideonah@ncc.ng,2 cosmas.ani@unn.edu.ng
}

\begin{abstract}
Computer simulation modelling has the advantage of flexibility and modelling accuracy. However, it has limitations in its ability to be used to simulate cell loss rate when deriving the optimum resources required for data services to guarantee specific Quality of Service (QoS) requirement. Cell loss rates are simulated with excessive and unacceptable computer simulation run times. This limitation was overcome in an earlier publication by the author using an integrated simulation technique. This paper, therefore, describes the application of the integrated simulation technique for deriving the optimum resources required for data services in an asynchronous transfer mode (ATM) based private wide area network (WAN) to guarantee specific QoS requirement. The simulation tool drastically cuts the simulation runtime and is much more accurate. The effectiveness of the technique with data services is demonstrated by the simulation of an ATM switching node and in comparison with traditional approach.
\end{abstract}

Keywords: Asynchronous transfer mode node; integrated modelling; simulation; Cell loss.

\section{Introduction}

It has been shown that computer simulation modelling has the advantage of flexibility and modelling accuracy [1]. Modelling shared buffer system using analytical modelling technique is complicated since the queues in the buffer are strongly dependent [2]. However, advantage notwithstanding, computer simulation modelling technique has limitations in its ability to calculate cell loss rate with low value. For instance, a cell loss rate of less than 1E-6 clearly cannot be successfully calculated without involving excessive and unacceptable computer simulation run time. This is particularly serious in a developing environment such as in Nigeria where power supply is erratic. Cell loss rate is often recorded as zero where enough cells cannot be generated, within a computer run time, to enable such a low cell loss rate value (for instance, 1E-6) to be evaluated. It is obvious that resource allocation decisions made in those regions cannot be right. A computer simulation modelling approach which integrates mathematical and computer simulation modelling techniques solves the problem by switching from computer simulation to mathematical (analytical) modelling when enough cells cannot be generated for accurate cell loss evaluation [1, 3, 4]. The switching point between the two methods is determined by the maximum buffer storage value. The simulation tool is much more accurate. With the integrated method, cell loss rate is predicted in those regions where computer simulation records a zero cell loss. The integrated simulation technique provides a good insight into the overall performance of a complete ATM network as it is able to predict a very low cell loss rate to a good level of accuracy. Moreover, the approach is applicable in a wide range of application environments involving rare events. The effectiveness of the technique with data services is demonstrated in relation to the simulation of an ATM switching node and in comparison with traditional approach.

The approach is used to quantify the cell loss rate for data traffic that is loss sensitive with a very low value of cell loss rate standard's requirement. This technique has been applied successfully for video related services [3]. Therefore, this work is a part of an on-going research work in the Centre (Centre of Excellence in Networking) on the performance management of an ATM based enterprise-wide network. By applying the same methodology as in video related services, 
transmission bandwidth and buffer resources are more accurately determined for a given homogeneous data traffic load and specific QoS requirement. A comparison is made between this technique and the traditional method to substantiate the need for the application of the integrated simulation method.

\section{Integrated simulation model}

Figure 1 shows the integrated simulation model. It differs from the traditional approach with the inclusion of a cell loss rate predicting module and the associated arrival and departure process monitors. The cell loss rate predicting module is an analytical model based on a mathematical expression which is developed using the fluidflow approximation approach [1,3]. The arrival and departure processes are monitored using rate monitor [3].

\subsection{Cell loss predicting module}

The predicting module is an analytical model that has the objective of predicting cell loss rate in those regions where the use of computer simulation modelling is impractical. Using the module, the performance of the switching node is evaluated for specified traffic loads. The above objective is achieved by evaluating the probability of cell(s) arriving at the transmission buffer queue when the queue is full.

The analytical model is simply a mathematical expression developed by applying queuing theory. The process of cell arrival at the queue is characterised by the same parameters used in developing the source model in terms of peak rate, $\pi$, mean rate, $\lambda$, and average burst duration, $\tau$. The cell departure rate is characterized by the rate at which the queue is served, $\mu$, which in turn is determined by the bandwidth of the link. The queue has a finite buffer capacity, $\kappa$.

The Markov chain two-state model has been used by a number of researchers to evaluate the QoS of an ATM network $[5,6,7,8]$. The use of this approach to evaluate the probability of cell loss involves a complex system of differential equations that have not got explicit solutions and therefore must be computed numerically [6]. In order to simplify the mathematics and the corresponding computations involved in the evaluation - without loosing the precision required to obtain satisfactory performance results - the fluid flow approximation method is used on the Markov chain. The method assumes a uniform service and arrival process - continuous information flow - instead of the discrete flow of cells $[7,8,9,10]$. Fluid flow approximation compares favourably with other existing and popularly accepted methods [8, 11]. Without going into the rigour of deriving and solving the system of differential equations involved in this method, existing and appropriate expressions developed through the same approach for single and multiple sources are employed $[8,9]$.

The time interval between cell arrivals and cell departures are monitored during a simulation run at the points indicated in Figure 1 . The average time between cell arrivals, $\alpha$, and cell departures, $\beta$, are measured for a constant time period. The traffic descriptors can be expressed as a function of $\alpha, \beta$, and the queue service rate (and hence link bit rate), $\mu$, using the following expressions:

$$
\begin{aligned}
& \lambda=\frac{1}{\text { mean value of } \alpha} \\
& \pi=\frac{1}{\text { Minimum value of }} \alpha \\
& \mu=\frac{1}{\text { Minimum value of } \beta} \\
& \text { Ts }=\frac{\sum \alpha^{\prime}}{\text { Total number of } \alpha^{\prime}} \\
& \tau=\frac{\lambda * \text { Ts }}{\pi-\lambda}
\end{aligned}
$$

where $\lambda$ is the mean cell rate, $\pi$ is the peak cell rate, Ts is the average silence duration and $\tau$ is the average burst duration. $\alpha^{\prime}$ is an average cell inter-arrival period that is greater than $1 / \lambda$ and is established by simulation. The parameter $\kappa$ shown in Figure 1 is the maximum buffer occupancy and is expressed in units of 53 bytes (cell size). The queue is serviced using a First-In First-Out (FIFO) queuing priority strategy at the rate of $\mu$.

The model is translated into a Designer executable model that is compatible with the computer simulation model of figure 1 using the designer BDE primitives [12].

\section{Simulation results}

The integrated model in Figure 1 was simulated and the results shown in Figures 2 - 5. Validation of the integrated modelling approach has been carried out based on a single and multiple data traffic sources [3]. 


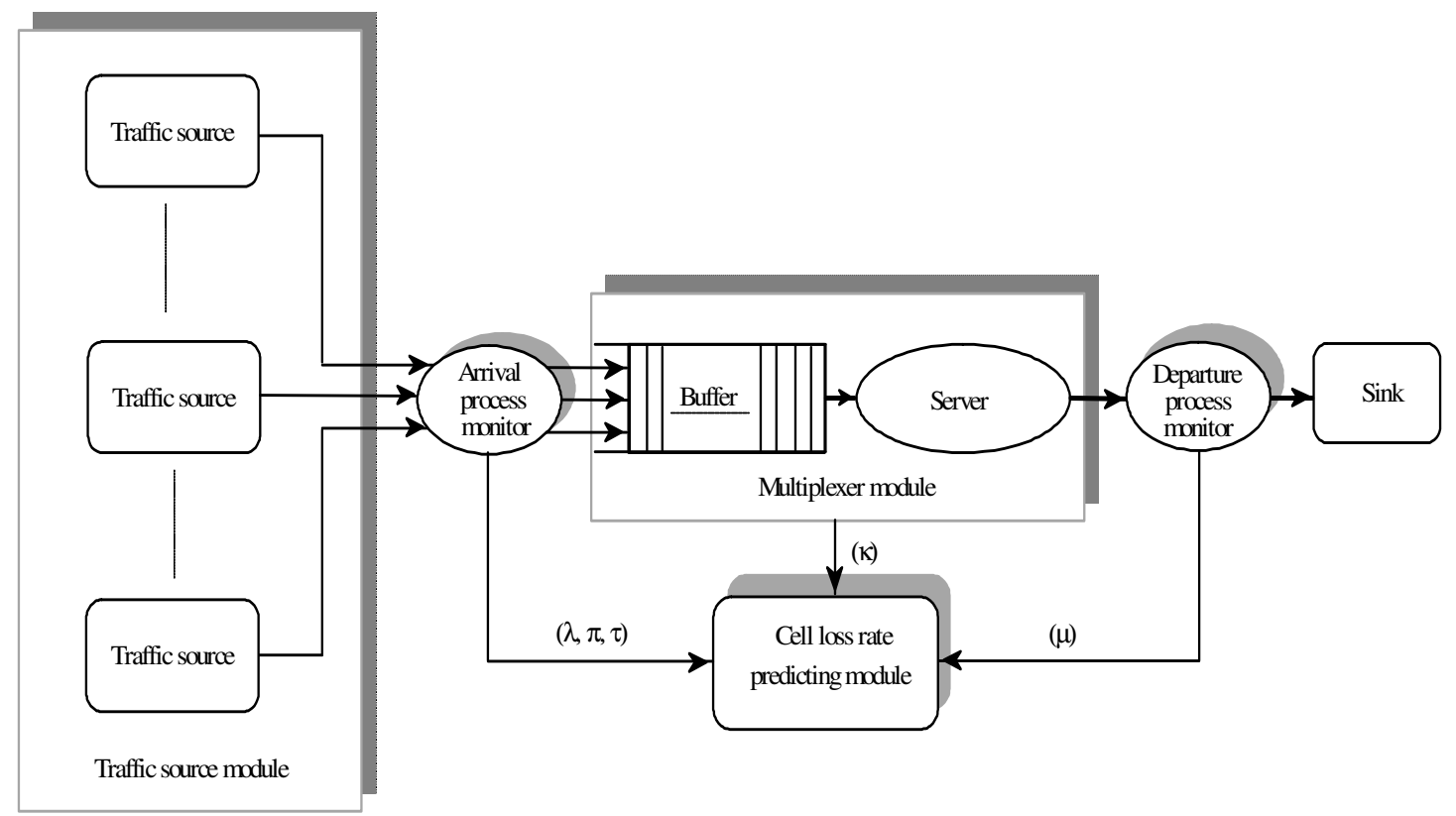

Figure 1: Integrated simulation model

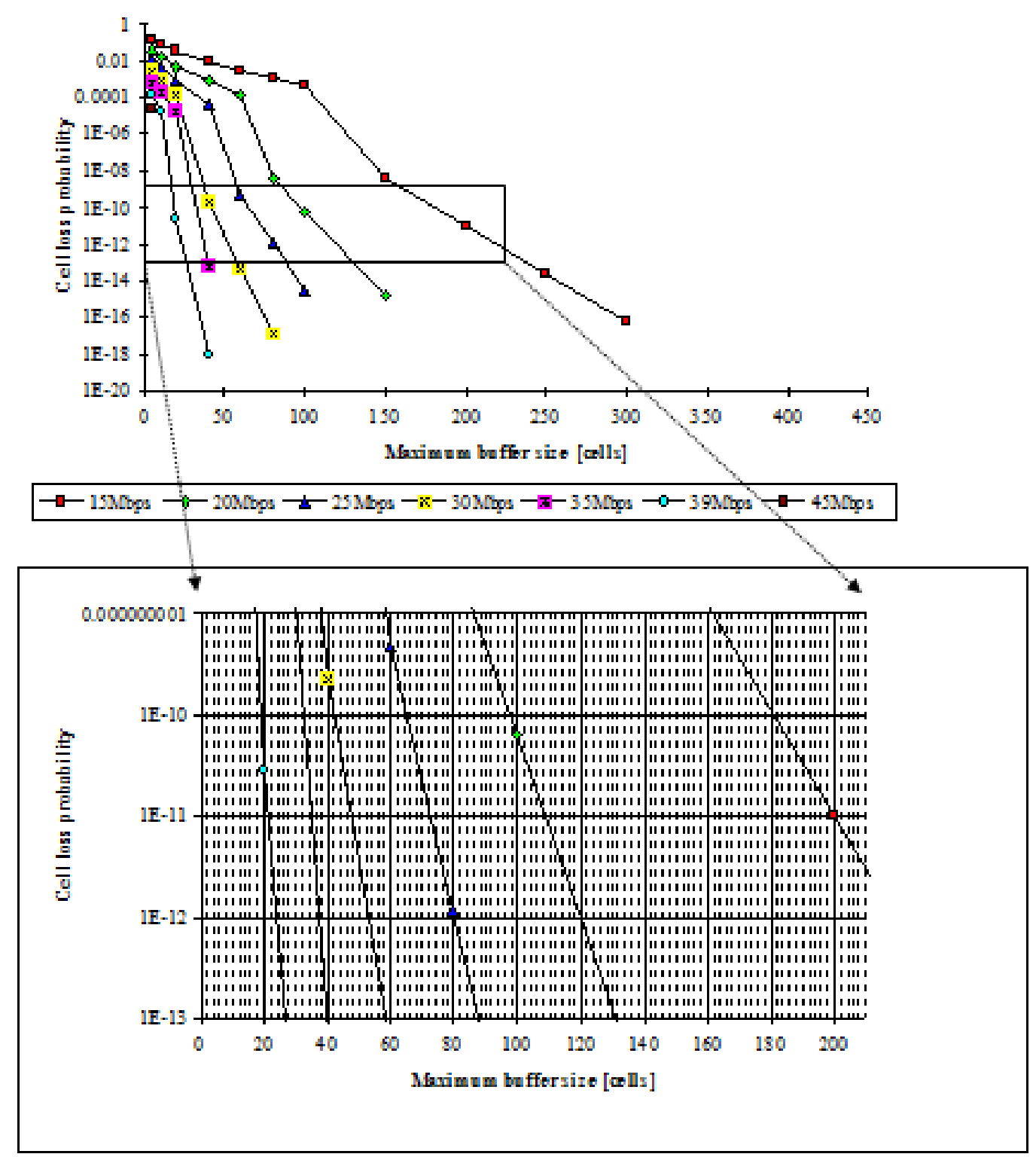

Figure 2: Data traffic cell loss rate as a function of buffer size at various bandwidths for 5 sources 
The integrated model is comprised of Designer model which was simulated for data sources and the cell loss predicting model. Cell loss probability is computed using the same condition as in the case of video related services [3]. The traditional model was simulated under the same condition and parameter values as in the case of integrated model. The results obtained from the traditional computer simulation model were compared with those produced by the integrated computer simulation model [1]. The integrated model evaluates cell loss rate using the arrival and departure processes monitored and characterised by the same parameters used in both computer simulation and analytical models but translated to match the representations earlier described. The cell loss probability was evaluated under the same conditions as in the computer simulation case for both single and multiple sources.
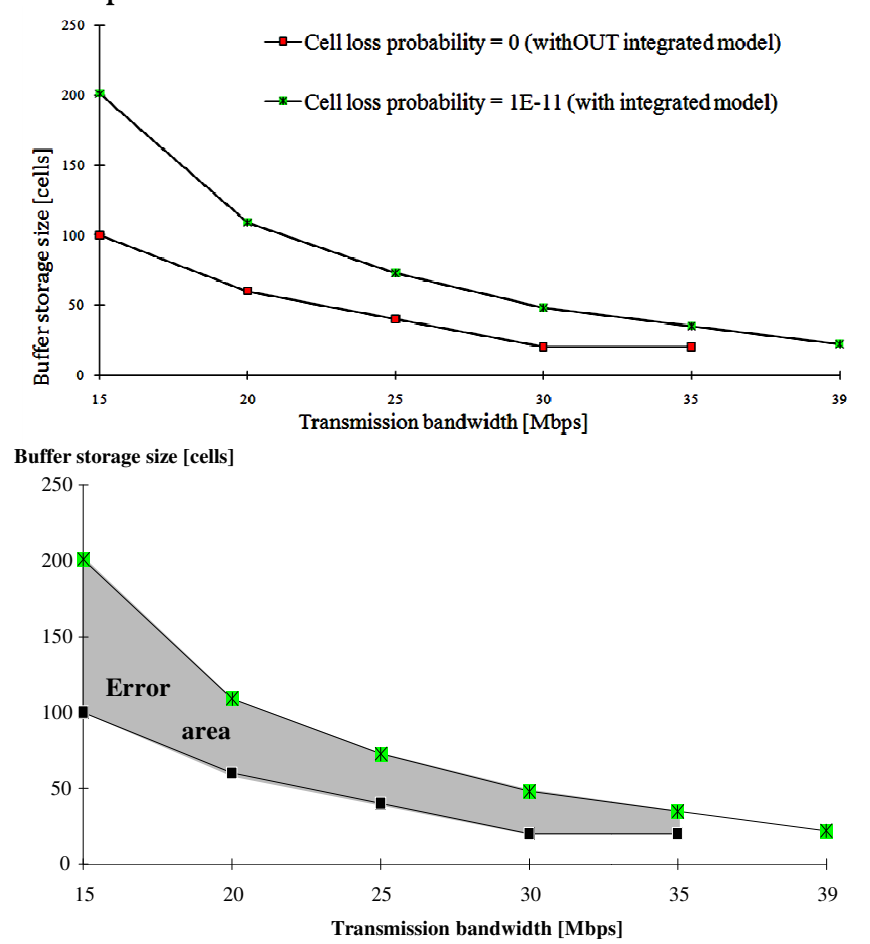

Figure 3: Comparison between integrated and traditional simulation model for data traffic sources $=5$

Figure 2 illustrates the relationship between cell loss rate and transmission buffer capacity at varied values of link bandwidths of 15 - $45 \mathrm{Mbps}$ for single and multiple sources. The results are very similar for single and multiple sources (1 20 sources). Therefore, the case for only 5 sources is presented for illustration in order to avoid duplication. The curves show that a low value of cell loss rate can be estimated from the probability of cell loss obtained using the integrated computer simulation model. Figure 2 shows that the cell loss probability relationship has the same pattern as those obtained using traditional method and only differs with the extension of the curves down to very low cell loss values. The curves also show that increase in buffer size results in a decrease in the value of cell loss rate.

The relationships shown in Figure 3 were derived from the curves obtained from the quantification of cell loss rate using the traditional and the integrated simulation modelling approaches. Figure 3 shows the relationship between buffer storage size and transmission bandwidth for traditional computer simulation model and that for integrated computer simulation model. The models were simulated for cell loss rate equal to zero and 1E-11 respectively. Both curves show that reduction in buffer size necessitates increase in the link transmission bandwidth for a given cell loss rate (QoS) and sources (traffic intensity).

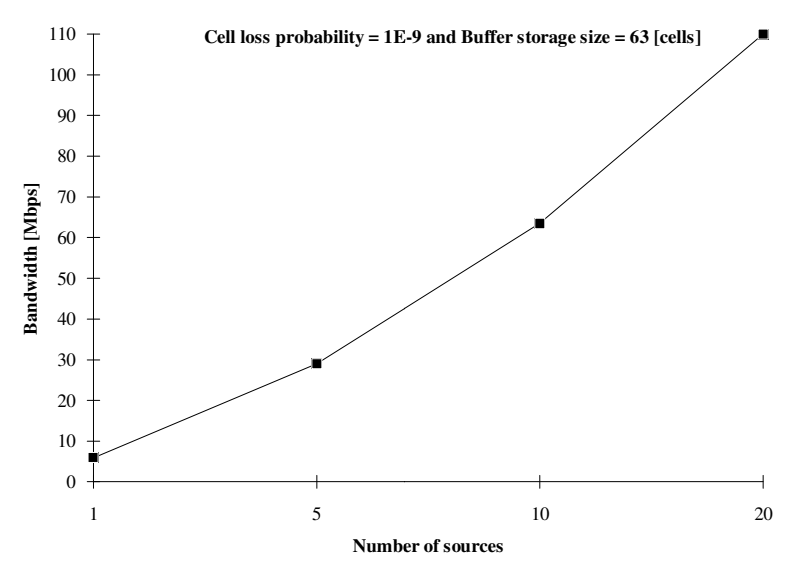

Figure 4: Data traffic resource allocation curve

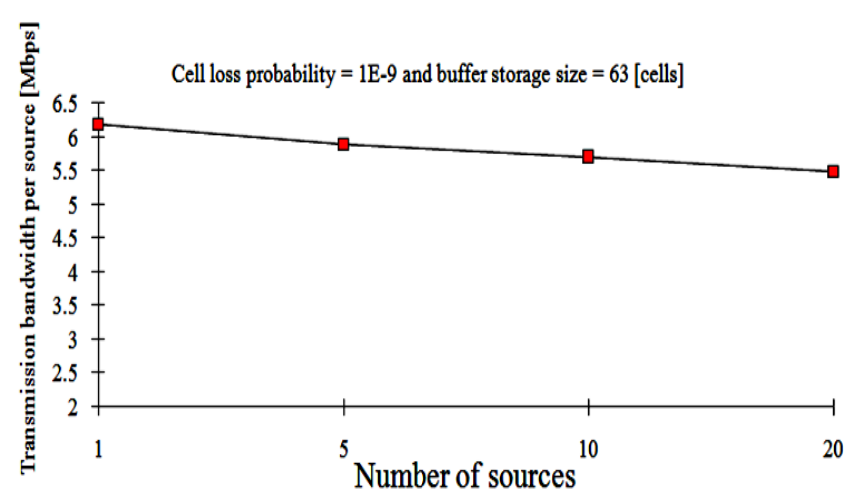

Figure 5: Statistical multiplexing gain

More importantly, the curves in figure 3 are used for the comparison between the traditional and the integrated computer simulation approach. The error area between the two curves illustrates the disparity between the two approaches. In 
practice, using the traditional simulation method for the analysis of cell loss rate as low as $1 \mathrm{E}-11$ will result in resource allocation decision being taken in the error area. Using the traditional approach, it was possible to generate 100,000 cells within 24 hours with which analysis on cell loss rate were conducted. This implies that cell loss rate below $1 \mathrm{E}-5$ cannot be obtained within 24 hours of non-power outage. In our environment (Nigeria) this is a very difficult situation. The model records any order cell loss rate value below $1 \mathrm{E}-5$ as zero. This is therefore, the practical explanation of the reason behind the disparity mentioned above. The practical implication is that if one is required to run a private ATM based network that should conform with $1 \mathrm{E}-11$ cell loss rate (QoS) using 80-cell transmission buffer capacity; there will be the need to lease an optimal (least) transmission link bandwidth to satisfy the condition. Using the integrated simulation model, the given QoS would require precisely $24 \mathrm{Mbps}$ transmission link bandwidth while if it were the traditional model it would indicate $17.5 \mathrm{Mbps}$ link bandwidth. Though the 17.5 Mbps bandwidth may appear more attractive, the network will operate much below the recommended $1 \mathrm{E}-11$ cell loss rate (QoS).

Figure 4 shows the data traffic resource allocation curve; and it illustrates the amount of bandwidth required for each of the different number of sources given a cell loss rate of $1 \mathrm{E}-9$ and buffer storage capacity $=63$ cells. Similar graphs can be derived from the results presented in Figure 2 for other specific cell loss probability values and buffer storage sizes. Figure 4 can be used to determine the effective number of homogeneous data sources that a given leased circuit can support without compromising the specified QoS. The curve can also be transformed into an analytical expression (power series) which can be more readily used by a resource management system.

Figure 5 shows that with an increase in the number of sources, the required bandwidth per source decreases. The curve thus illustrates the variation in statistical multiplexing gain that is obtained.

\section{Conclusion}

This paper has presented the application of integrated simulation technique to resource allocation to support data services in an ATM based private WAN. The simulation modelling technique is based on the integration of computer simulation and mathematical modelling methods. The technique has been proved particularly useful for quantifying rare-event occurrences such as cell loss rate in ATM networks.

This paper has effectively presented the approach that may be used to determine the effective and efficient number of homogeneous data sources (of a given traffic intensity) that specific network resources (transmission link bandwidth and buffer capacity) can support without compromising cell loss rate standard. In order words, the method with which optimal network resources may be allocated to data services for specified QoS is presented in Figure 4. The curves determined using this method can be transformed into an analytical expression (power series) which can be more readily used by a resource management system. The statistical multiplexing technique employed provided visible multiplexing gain as illustrated in Figure 5. The integrated technique was compares with the traditional approach and the disparity between then highlighted as illustrated in Figure 3 . It is therefore very clear that any resource allocation decision made with the traditional simulation technique is vulnerable to error.

\section{Acknowledgement}

The authors are grateful to other Networks Research Team members for their constructive comments. Authors are grateful to the University of Nigeria, Nsukka for providing the enabling environment and the Federal Government of Nigeria for endowing the University with Centre of Excellence in Networking that made this work possible.

\section{References}

1. Ani C. I. and Halsall F., "Simulation Technique for evaluating Cell-Loss Rate in ATM Networks"; The Society for Computer simulation, SIMULATION Journal, California; Vol. 64, No. 5; May 1995; pp. 320-329.

2. Zhang Z. and Yang Y., "A Novel Analytical Model for Electronic and Optical Switches with Shared Buffer"; IEEE/ACM Transactions on Networking, Vol. 15, No. 5, October 2007.

3. Ani C.I., Ahmad R. and Halsall F., "Methodology for the derivation of network resources to support video-related services in ATM based private wide 
area networks"; IEE Proc. Communication Vol. 142, No. 4, August 1995.

4. Mom J. M. and Ani C. I., "An Integrated BlockOriented Simulation Model for Estimating Cell Loss Rate in ATM Networks" Pacific Journal of Science and Technology. Volume 13. Number 1. May 2012, pp. 287-291

5. Anick D., Mitra D. and Sondhi M. M., "Stochastic Theory of a Data-Handling System with multiple Sources", The Bell System Technical Journal, October 1982, USA, pp 1871 - 1894.

6. Roch Guerin, Hamid Ahmadi and Mahmoud Naghshineh, "Equivalent Capacity and its Application to Bandwidth Allocation in High Speed Networks" IEEE Journal on Selected Areas in Communications. Vol. 9, No. 7, September 1991 pp.968-981.

7. George Kesidis, Jean Walrand and Cheng-Shang Chang, "Effective Bandwidth for Multiclass Markov Fluids and Other ATM Sources", IEEE/ACM Transactions on Networking vol.1, No.4, August 1993.
8. Sykas D, et. al., "Congestion Control - Effective Bandwidth Allocation in ATM Networks", High Performance Networking, IV (C-14), IFIP, Belgium, 1993, pp.65-80.

9. Bijan Jabbari and Ferit Yegenoglu, "An efficient Method for Computing Cell Loss Probability for Heterogeneous Bursty Traffic in ATM Networks", International Journal of Digital and Analog Communication Systems, vol.5, 1992, 39-48

10. Gordon B. Agnew and Jon W. Mark, "Performance Modeling for Communications Networks at a Switching Node", IEEE Transactions on Communications vol. Com-32, No. 8, August 1984.

11. Frost V.S. and Melamed B., "Traffic Modelling For Telecommunications Networks", IEEE Communications Magazine, Vol. 32, No. 3, March 1994

12. COMDISCO SYSTEMS, "BONeS (Block Oriented Network Simulator) Designer", A business unit of Cadence Design Systems, Inc., USA, 1993 